\title{
Germanica
}

GERMANICA

$61 \mid 2017$

Le conte comme esthétique et stratégie du détour dans la littérature et le cinéma

\section{Wie ich mir das Glück vorstelle de Martin Kordic et l'esthétique du conte}

Wie ich mir das Glück vorstelle von Martin Kordic und die Ästhetik des

Märchens

Wie ich mir das Glück vorstelle from Martin Kordic and the aesthetics of tale

\section{Emmanuelle Aurenche-Beau}

\section{(2) OpenEdition}

Journals

Édition électronique

URL : http://journals.openedition.org/germanica/4039

DOI : 10.4000/germanica.4039

ISSN : 2107-0784

Éditeur

Université de Lille

Édition imprimée

Date de publication : 31 décembre 2017

Pagination : 61-72

ISBN : 9782913857407

ISSN : 0984-2632

Référence électronique

Emmanuelle Aurenche-Beau, « Wie ich mir das Glück vorstelle de Martin Kordic et l'esthétique du conte », Germanica [En ligne], 61 | 2017, mis en ligne le 01 janvier 2021, consulté le 03 mars 2021

URL : http://journals.openedition.org/germanica/4039; DOI : https://doi.org/10.4000/germanica.4039

(c) Tous droits réservés 


\title{
Wie ich mir das Glück vorstelle de Martin Kordic et l'esthétique du conte
}

\author{
Emmanuelle AURENCHE-BEAU \\ Université Lumière Lyon 2
}

En 2015, un des « Förderpreise » du Prix Adalbert von Chamisso qui récompense chaque année, comme on le sait, un texte écrit par un auteur « dont l'œuvre est marquée par un changement de culture » et par

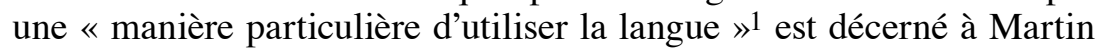
Kordic pour son premier roman, Wie ich mir das Glück vorstelle ${ }^{2}$. Né d'une mère allemande et d'un père croate originaire d'un village situé près de Mostar en Bosnie-Herzégovine ${ }^{3}$, il a, comme il l'explique dans une interview, éprouvé la nécessité de se confronter au thème de la guerre, un thème omniprésent au sein de sa famille : ses parents, installés en Allemagne où il est né en 1983, ont en effet accueilli leur

1. - http://www.bosch-stiftung.de/content/language1/html/58869. asp?version=mobile

2. - Le livre a été publié en 2014 au Hanser Verlag. Nous citerons ici l'édition Fischer (Frankfurt a. M., 2015) et indiquerons uniquement le numéro de la page entre parenthèses lorsque nous le citerons.

3. - Le critique Richard Kämmerlings précise : «Martin Kordić wurde 1983 in Celle geboren und ist in Ludwigshafen/Mannheim aufgewachsen. Seine Mutter ist Deutsche, sein Vater ein kroatischer Gastarbeiter aus Bosnien. Die Familie kommt aus einem kleinen Dorf in der Nähe von Mostar, auf halbem Weg nach Medjugorje, einem bekannten Marienwallfahrtsort ». https://www.welt.de/kultur/literarischewelt/ article124479857/Seit-ich-denken-kann-war-dieser-Stoff-da.html. 
famille et leurs proches fuyant le conflit qui faisait rage en Bosnie et il a, enfant et adolescent, entendu leurs histoires - «la matière était là $»^{4}$.

La matière était là, mais il fallait trouver une forme à même de restituer au plus juste ces expériences indicibles. Après de nombreuses tentatives infructueuses entre autres du côté du roman de famille ou du roman de génération (l'auteur a mis cinq ans à écrire le livre) ${ }^{5}$, Kordic a opté pour une langue et pour une écriture qui ont pu être rapprochées du conte : le jury du prix Chamisso souligne ainsi le contraste entre « une langue prétendument naïve, se rapprochant à certains moments de celle du conte », également qualifiée de " poétique », et « les événements d'une grande inhumanité » évoqués par le narrateur ${ }^{6}$, tandis que le critique du Deutschlandfunk note que l'écriture de Kordic est à même de «conjurer» l'horreur d'un monde qui « dépasse le narrateur» et résume le livre par la formule: « un apprivoisement de l'incompréhensible à la manière d'un conte $»^{7}$.

Après avoir analysé la manière dont l'auteur donne à voir l'irruption et la réalité de la guerre dans la vie de son personnage principal, le jeune Viktor, nous étudierons les aspects qui peuvent apparenter au conte le récit de sa vie et notamment de sa survie dans la ville dévastée, tout en nous interrogeant sur la présence d'autres références littéraires au sein du livre qui a également reçu la médaille Alfred Döblin.

4. — Ibid.: «Während der Balkan-Kriege Anfang bis Mitte der Neunziger flohen viele zu ihren Verwandten ins Ausland; auch bei den Kordićs kamen Tanten, Cousins und Cousinen unter. Der Krieg gehörte zur Kindheit. Martin Kordić sagt: „Seit ich denken kann, ist das Thema. Es war immer da, weil meine Verwandten da waren." Schon seine ersten Prosaversuche mit siebzehn, achtzehn kreisten darum. „Dieser Stoff war in meinem Schreiben bereits da, bevor ich überhaupt wusste, was Gegenwartsliteratur ist." ».

5. - Ibid., Selon Richard Kämmerlings qui a interviewé Martin Kordic : «Lange hat Kordić nach der richtigen Form für diesen Lebensstoff gesucht. Frühere Versuche, so erzählt er, seien auf einen Familien- oder Generationenroman hinausgelaufen ».

6. - « Viktors vermeintlich naive, bisweilen märchenhafte Sprache steht in krassem Gegensatz zu den oft extrem inhumanen Geschehnissen, von denen er berichtet. Diese behutsame poetische Sprache macht die literarische Besonderheit des Romans aus ». http://www.boschstiftung.de/content/language $1 / \mathrm{html} / 58869$.asp? version=mobile

7. - « Der Roman „Wie ich mir das Glück vorstelle“ ... zeichnet das Bild einer auf vielen Ebenen heillosen Welt. Alles Erlebte wird am Rande der Sprache brüchig, weil es von Verwüstung erzählt, die einen Jungen - den Erzähler - überfordert. Doch zugleich bannt er dadurch, dass und wie er erzählt, den Schrecken und die Trauer, ja er verleiht ihr sogar Humor und berührenden Glanz: Eine märchenhafte Zähmung des Unverständlichen - und ein wirklich beeindruckendes Debüt. » http://www.deutschlandfunk.de/martin-kordic-wie-ich-mir-das-glueck-vorstelle-vom-elend.700.de.htm1?dram:article_id=296920. 


\section{L'irruption et l'évocation de la guerre}

Si le roman ne livre pas un récit linéaire de la vie de son " héros", mais alterne au contraire des chapitres de longueurs variées correspondant à différents moments et à différents lieux de sa vie, il est néanmoins possible d'y distinguer trois phases: la période qui précède la guerre; son séjour dans un orphelinat; le temps de sa survie dans la ville jusqu'au « dernier jour » où il la quitte après la disparition de tous ses compagnons de survie.

La première période est présentée comme une période heureuse. Viktor vit avec ses parents et son frère dans une ville multiculturelle qui n'est pas nommée (mais dans laquelle on a pu reconnaître Mostar et ses ponts) où les relations entre communautés semblent aller de soi. Bien qu'appartenant à la communauté des « Kreuzer », sa famille habite sur la rive droite du fleuve dans un quartier habité en majorité par des « Mudschis $»^{8}(87)$. Dans son immeuble, il y a huit familles « Kreuzer» et vingt-deux familles « Mudschi » (87). Son frère est ami avec un jeune « Mudschi » et sa mère est également amie avec la mère du garçon en question (87). Son père est souvent en déplacement - il travaille « dans le désert » où il creuse « des trous » pour en retirer « un trésor » (80), sans doute du pétrole. La famille semble vivre dans une certaine aisance ainsi qu'en témoigne la description de leur appartement (104). Lété, il passe ses vacances chez ses grands-parents au «village des gens heureux ». Il y joue avec ses cousins et a une grande complicité avec sa grand-mère qui lui a sauvé la vie au moment de sa naissance (21).

Les «dernières vacances » sont cependant marquées par l'irruption de la mort et de la violence. Un enterrement a lieu dans le village (48); il trouve un fusil sous un lit (51); un tank s'approche de la maison de sa grand-mère, un homme en descend, entre sans enlever ses chaussures, fouille toutes les pièces, trouve le fusil, puis un coussin rempli de grenades (52) et les maudit tous (53).

Son premier jour d'école coïncide avec la fin du «pays de tous les peuples » (la Yougoslavie), ainsi que l'annonce «l'homme de la télévision ». Son père ne travaille pas, il a été renvoyé chez lui. La télévision montre un tank en train d'entrer dans un village (65). Peu après, son père reçoit un uniforme et un fusil (66) et part combattre. Il le voit peu à peu changer, devenir de plus en plus fanatique (93), interdisant à sa femme et à ses fils de fréquenter les voisins «Mudschi » (86). Les tensions entre communautés s'exacerbent, les enfants «Mudschi » changent presque tous d'école (88), un des enfants «Mudschi » qui est encore

8. - Nous avons fait le choix dans notre traduction de garder les termes allemands qui désignent vraisemblablement l'un les chrétiens, les Croates, l'autre les musulmans, les Bosniaques, l'important étant de toute façon l'opposition entre deux communautés. 
dans sa classe est contraint par le maître («Kreuzer ») de cracher sur la vitrine du magasin (« Mudschi ») où travaille sa mère (90). Les enfants se mettent à jouer à la guerre (110), maltraitent un enfant « Mudschi » (111).

Un jour, la grand-mère, les tantes et les cousins de Viktor (soit en tout treize personnes) arrivent chez eux, fuyant les combats qui font rage dans le village (103). La vie se réorganise, à seize dans l'appartement - ils sont même filmés par un journaliste qui commente la situation en expliquant que la ville « est menacée par le même sort que les villages »: « les réfugiés transfèrent le traumatisme du Grand Combat dans la ville, ils la divisent $»$. Quelque temps après, ils reçoivent l'ordre de partir et de passer de l'autre côté du fleuve (124): ils ont dix minutes pour rassembler leurs affaires, sont comptés, puis séparés en deux groupes et contraints de partir à pied dans la neige (128). Viktor cependant se rend compte qu'il a oublié de laisser dans la boîte aux lettres le mot qu'il a préparé pour son père (125), il quitte le groupe et rebrousse chemin.

Errant ensuite dans la neige, il est recueilli par des religieuses de la «communauté des enfants de Marie » qui l'emmènent dans un orphelinat situé sur une montagne appelée la «montagne du voyant» (122) où vivent d'autres enfants traumatisés par la guerre - l'un d'eux se suicidera en se jetant dans un puits. Après avoir été pendant un temps chargé de tenir le journal de l'institution, il se voit confier la tâche de vendre de l'eau aux pèlerins qui se rendent au sanctuaire marial situé au sommet de la «montagne des apparitions », mais ce travail est pénible sous une chaleur de plomb et il semble préférer passer son temps non loin de là, devant le magasin de «Bubka » (9) jusqu'au jour où il part, profitant de la voiture d'un couple de jeunes mariés qui, après avoir pris des photos, retourne à la «ville des ponts » (28). S'ouvre alors une nouvelle partie de sa vie où il tente de survivre dans la ville dévastée par la guerre en compagnie d'un adolescent infirme un peu plus âgé que lui et d'autres personnes rencontrées au hasard de ses déambulations.

\section{Le dispositif narratif}

Une partie des caractéristiques du style du roman s'explique à l'évidence par le choix du dispositif narratif. Le narrateur en effet n'est autre que Viktor lui-même qui écrit son histoire pour son compagnon de survie dans la «ville des ponts », le « Dschib unijambiste», dans un cahier que ce dernier lui a donné le jour de leur rencontre, mais le récit adressé à un « tu » est plus largement destiné « à celui qui le lira »10. Il

9. - « «ieser Stadt droht das gleiche Schicksal wie den Dörfern [...], die Flüchtlinge bringen das Trauma des Großen Kampfes mit in die Stadt, sie spalten sie. », p. 108.

10. - « Ich schreibe sie für den einen, der sie liest », p. 79. 
contient d'un côté l'évocation de l'enfance de Viktor depuis sa naissance jusqu'à son retour dans la ville après son séjour à l'orphelinat et d'un autre, à la manière d'un journal, le récit au jour le jour de sa survie dans la ville jusqu'au «dernier jour » qui coïncide avec la fin du cahier et la fin du livre (cela est souligné par la coïncidence rigoureuse entre les numéros des pages, cf. par exemple p. 79 et p. 115).

Le fait que le narrateur soit un enfant ou un jeune adolescent peut expliquer l'absence de contextualisation du récit: l'absence de dates, la présentation un peu naïve du conflit non situé géographiquement, appelé simplement le «Grand Combat » (58), la désignation non-historique des forces en présence les « hommes de la montagne » (85), les « Mudschis » (55), les « Kreuzer » $(55)^{11}$, les « guerriers des autres pays » (58), ainsi que l'absence d'explication sur les raisons de la guerre. Le récit de Viktor nous donne seulement à voir les conséquences de la guerre telles qu'il les perçoit. La campagne est ravagée: « Nous traversons quelques villages. Partout, il n'y a plus que deux ou trois maisons. Certaines n'ont plus de toit $»^{12}$. La ville est transformée en champ de décombres : «Du cimetière, nous arrivons directement aux rues de la ville. Quelques voitures renversées et partout une grande quantité de décombres. Cela sent les poules brûlées. L'air a une odeur de rouille et me fait mal à la gorge [...] Il n'y a pas de bruit. Il n'y a personne dans la rue » $(44)^{13}$, et les jardins sont transformés en cimetières ${ }^{14}$.

Si le texte de Viktor ne fournit aucun élément de contextualisation historique ou géographique (aucun des lieux ne porte de nom géographique, tous portent, au contraire, comme on l'a vu, des noms abstraits comme «le village des gens heureux », «la montagne du voyant», « la ville des ponts »), il ne contient pas non plus de description de son monde intérieur. Le lecteur ignore ses sentiments. Même lorsqu'il vit des événements terriblement douloureux pour lui comme lorsqu'il se fait dérober le « paquet » qui contient tout ce qu'il possède, il se contente de noter: «Je laisse mon paquet et m'en vais » ${ }^{15}$. Traumatisé par tout ce qu'il a vécu, il a probablement mis en place des mécanismes de défense pour se protéger de tout ressenti et se limite au récit des faits ${ }^{16}$. Il en va

11. - Qui peuvent représenter les Serbes, les Bosniaques et les Croates.

12. - «Wir kommen durch ein paar Dörfer. Da sind überall noch zwei oder drei Häuser. Manche haben keine Dächer mehr », p. 29.

13. - « Vom Friedhof aus kommen wir direkt in die Straßen von der Stadt. Ein paar umgestürzte Autos und jede Menge Schutt liegt hier rum. Es riecht nach verbrannten Hühnern. Die Luft schmeckt nach Rost und tut mir im Hals weh. [...] Es ist still. Keiner ist auf der Straße », p. 44 ; cf. aussi p. 56.

14. - «Jeder Garten ist jetzt ein Friedhof», p. 55.

15. - « Ich lasse das Paket stehen und gehe weg », p. 44.

16. - Il ne semble pas non plus éprouver d'émotion particulière quand il retrouve son « paquet» $(60)$. 
de même, peu de temps après, lorsqu'il sauve involontairement la vie à celui qui lui a pris son paquet et qui a lui-même été jeté dans le fleuve par trois hommes qui lui ont, à leur tour, volé tout ce qu'il possédait. Il le raccompagne chez lui et conclut laconiquement: «C'est là que j'habite aussi maintenant ${ }^{17}$. Vivant dans la ville en guerre, habitué peut-être à la violence quotidienne qui y règne, il ne semble connaître ni l'étonnement, ni la peur quand il est réveillé par le bruit d'une violente explosion (40), quand il manque de sauter sur une mine (41) ou quand il est menacé par le revolver de son agresseur (43-44). La disparition successive de tous ses compagnons de survie qu'il a soignés et à qui il semble pourtant s'être attaché ne donne pas lieu non plus à un commentaire particulier; sa réaction, quand il comprend que plus rien ne le retient dans la ville, est de partir en emportant son cahier et son crayon dans un sac en plastique (150).

Un autre trait frappant de l'écriture de Viktor/Kordic étroitement lié à ce style très factuel est la parataxe, la simple juxtaposition de notations, sans institution de lien logique. Le récit se présente comme une succession d'épisodes qui coïncident avec les rencontres que fait Viktor ${ }^{18}$. Il est centré aussi sur le combat quotidien pour la survie : manger - se rendre à la soupe populaire en traversant la ligne de démarcation (56-59), tuer des oiseaux qui s'avèrent immangeables (96), accepter d'être interviewé par un journaliste pour un repas au restaurant (76), faire la plonge dans un bouge pour pouvoir manger les restes laissés par les clients (96) - et boire, se laver, laver ses vêtements sans être contaminé par l'eau polluée en raison des cadavres jetés dans le fleuve (94), pouvoir se mettre à l'abri et dormir dans un lieu relativement sûr, piller des tombes pour récupérer des objets susceptibles d'être revendus $(76)^{19}$.

\section{Esthétique du conte}

La tendance à l'abstraction qui vient d'être évoquée à propos du traitement de l'espace et du temps ainsi que le refus de la psychologie et le style paratactique font sans doute partie des éléments majeurs qui expliquent que le livre a pu être rapproché du conte. Certains éléments de la vie de Viktor peuvent en outre rappeler certains contes populaires. Le récit quelque peu extraordinaire de sa naissance (Viktor ne doit sa survie qu'à l'intervention hardie de sa grand-mère à laquelle on pourrait attribuer le rôle de bonne fée ${ }^{20}$ ), ainsi que la particularité physique,

17. - « Hier wohne jetzt auch ich », p. 46.

18. - Un certain nombre des chapitres qui sont consacrés à sa vie dans la « ville des ponts » portent comme titre le nom des personnes qu'il rencontre : «Der einbeinige Dschib » (p. 40), «Unser Mädchen » (p. 94), « Die Alte» (p. 112).

19. - Viktor fait à plusieurs reprises la liste de leur butin (p. 71, 77, 79).

20. - Dans la lettre qu'elle lui écrit, sa grand-mère fait allusion à l'aide qu'ils 
l'infirmité qu'il en garde (Viktor naît « tordu » et doit porter un corset), l'apparentent à certains héros de contes dont le handicap s'avère être le signe d'un destin exceptionnel - son prénom est choisi par sa grandmère et associé par elle à «la ville de l'or et des chercheurs d'or en Amérique » (22). Il en va de même pour ce que l'on pourrait appeler sa destinée: après une enfance heureuse, il quitte sa famille et se retrouve livré à lui-même, isolé au sein d'un monde dévasté dans lequel il n'a même plus de nom. Devenu par la force des choses ce que Lüthi appelle un « Wandernder » 21 , il passe d'un lieu à l'autre, quitte le monde clos de l'orphelinat pour le monde ouvert de la ville qu'il quitte à son tour.

Sa vie est rythmée par des rencontres de hasard 22 : celle déjà évoquée avec le Dschib qui, s'il est présenté d'abord comme un adversaire qui lui dérobe tout ce qu'il possède (43) (la ville est une jungle...), s'avèrera être une sorte de double (il est lui aussi infirme) dont il devient inséparable; celle avec le bébé abandonné et avec la fille "aux cheveux rouges » qui lui permettent d'apparaître comme un sauveur, protecteur des enfants et des femmes en danger; celle enfin avec la « vieille femme » mystérieusement en contact avec sa grand-mère dont elle lui confie une lettre. Notons en outre que la manière dont ces personnages sont décrits par Viktor en fait des êtres mi-humains, mi-fantastiques. Le Dschib ressemble à un gnome mi-enfant, mi-vieillard, mi-animal ${ }^{23}$ : «Le garçon a la peau noire et les cheveux gris. Ses cheveux poussent même sur son front qui est large et bas. Ses yeux ne regardent nulle part. Son visage est tout ridé. Le garçon a les cheveux et le visage d'un grandpère et la peau d'un homme du désert » (42). Il dit aussi de lui qu'il a « la force d'un magicien » et qu'« avec sa logorrhée bizarre et toutes les bêtises qu'il raconte, il lui fait une impression d'inquiétante étrangeté », et de conclure: " il croit aussi aux sorcières » (61). La fille aux cheveux rouges, dont il entend les cris derrière la porte de la cabane en bois dans laquelle elle se fait violer, est d'abord prise par lui pour un chien (98). Quant à la vieille femme qui en a peut-être trop vu dans sa vie, elle ressemble à une sorte de sorcière: «Elle n'a pas d'yeux. Son visage n'est fait que de peau. Son front va jusqu'aux joues » (119). Comment mieux faire ressentir les ravages de la guerre que par ces personnages déformés, à la limite de la monstruosité?

auraient reçue, lors de sa naissance difficile, de la part de celle qui est aussi celle qui lui transmet la lettre (la mystérieuse « vieille femme ») et que certains qualifient de sorcière et d'autres de femme aux pouvoirs surnaturels (p. 135).

21. - Max Lüthi, Das europäische Volksmärchen, Stuttgart, UTB, 2005 (1)̀̀re édition chez Francke en 1947), p. 29.

22. - La succession d'épisodes qu'elles engendrent est également un élément caractéristique du conte.

23. - « Der einbeinige Dschib singt wie ein kleiner Junge, obwohl er älter ist als ich und schon graue Haare hat », p. 96. 
Ces éléments mi-réalistes mi-fantastiques font eux aussi penser à l'univers du conte tout comme certains objets particulièrement précieux dont Viktor ne se sépare pas. Tout se passe comme s'ils avaient pour lui le pouvoir «magique » d'évoquer sa vie passée et de lui rappeler les personnes auxquelles il tient le plus: la photo que lui a donnée sa grand-mère qui le représente à trois ou quatre ans avec son frère (12), le morceau de bois que son père lui a rapporté du désert (19) et auquel il associe toute une histoire (80), la radio portative « Grundig Yacht Boy 500 » sur laquelle son père écoutait en boucle les informations et lui de la musique (83-85), et toutes sortes d'objets régulièrement énumérés dans le livre $(82,117)$ comme s'il s'agissait de talismans, dont il n'aura cependant plus besoin quand il aura terminé le cahier qui contiendra, sous une autre forme, l'ensemble de sa vie et de ses souvenirs. Lorsqu'il se retrouve seul - le Dschib et la vieille femme ont disparu (149) - il décide en effet de partir en n'emportant dans un sac en plastique que le cahier, le crayon et la lettre de sa grand-mère. Il met ses objets les plus précieux dans une boîte qu'il enterre (150). Davantage sans doute que la possession des objets, c'est en effet la mise en mots de sa vie qui permet à Viktor de survivre, associée au fait de raconter pour d'autres: comme il le dit lui-même, «si un jour, il n'y a plus d'histoire, si plus personne ne raconte, si plus personne n'écoute, c'est l'obscurité » (162163). On retrouverait ainsi une autre caractéristique du conte dont le propre est d'être transmis oralement, puis éventuellement par écrit. Or cette dimension de la transmission semble essentielle dans le livre.

\section{Conte et transmission}

Son histoire, ses histoires, Viktor les raconte en effet au Dschib et à la fille aux cheveux rouges: au Dschib, celle de sa naissance (20-22) qui devient son histoire préférée (79) et qui est précisément une histoire qui lui a été rapportée et qu'on reprend volontiers dans la famille parce qu'elle est extraordinaire ${ }^{24}$; à la fille aux cheveux rouges (101), celle des éléphants (67-68), celle de la première fois où il voit des éléphants en se rendant au zoo avec l'école 25 , celle de la «manière dont il s'imagine le bonheur » (165-170) et où il se voit précisément éléphant au sein d'une famille nombreuse qui ne se sépare pas (« Nous sommes nombreux. Nous restons toujours ensemble », 165). Or l'animal semble précisément, dans le livre, associé à la notion de chaîne de transmission reconstituée: chaque fois qu'il a écrit une page, Viktor dessine un éléphant sur le mur

24. - «Die Verwandten finden die Geschichte immer sehr spannend. Die Verwandten sprechen immer darüber, wenn ich in den Ferien zu Besuch bin », p. 20; « warum sich alle diese Geschichte später weitererzählen. », p. 21.

25. - Il s’imagine quittant le zoo avec un éléphant. 
de leur cabane. À la page 79 du livre qui est en même temps le cahier de Viktor, on lit: "Il y a maintenant soixante-dix-neuf éléphants et ils forment une chaîne de la trompe à la queue. » Or cette thématique de la transmission et de la réception des histoires est aussi celle du mystérieux prologue qui ouvre le livre.

Le livre débute en effet par un texte dont l'énonciateur est une personne dont on ne connaît ni le sexe ni l'âge. On sait seulement qu'elle habite seule « dans une des vieilles maisons, très haut dans la montagne », loin de toute civilisation ${ }^{26}$, une maison qui pourrait ressembler à celle de la photo qui clôt le livre, et qu'elle se nourrit de ce que lui apportent les gens qui montent jusqu'à elle. Mais les gens ne lui apportent pas seulement de la nourriture, mais aussi des histoires, les leurs ou celles qui leur ont été transmises par d'autres: « Parfois, j'entends même plusieurs fois la même histoire, avec de légères variations, tantôt, c'est l'un qui s'en sort mieux, tantôt c'est l'autre qui est le méchant, l'un raconte ce qu'il a vécu lui-même, l'autre rapporte ce qu'il a entendu d'un tiers » (7).

Or ce lieu hors du monde, en hauteur, pourrait rappeler le lieu que décrit la grand-mère de Viktor dans la lettre-testament qu'elle lui fait mystérieusement parvenir par la vieille femme avant de mourir : «Dans les montagnes. Tout en haut. Là où il n'y a pratiquement plus personne depuis longtemps déjà. Là où personne ne te demandera qui tu es. [...] C'est là-bas que tu dois aller. C'est là-bas qu'est ta place. Je serai avec toi. Regarde dans l'étable $»^{27}$. Tout se passe donc peut-être comme si la grand-mère, celle de Viktor, mais aussi celle de Kordic, lui assignait précisément la mission d'écouter et de transmettre à son tour ces histoires pour devenir lui-même et trouver sa place dans la chaîne des générations. Si le livre est dédié à « Baba Matija » (grand-mère Matija), c'est peut-être pour rendre hommage à celle qui est à l'origine du livre. On se souvient en effet, si l'on veut faire un parallèle avec Kordic lui-même, que l'écriture du livre est liée à la nécessité pour lui de s'approprier les histoires qu'il a entendues dans sa famille à propos de la guerre de Bosnie des années 1990.

\section{Autre référence intertextuelle}

Si de nombreux éléments du texte de Kordic peuvent donc l'apparenter au conte, il en est cependant un qui l'en distingue radicalement: le choix d'un narrateur à la première personne qui incite à chercher d'autres liens intertextuels. Or un certain nombre d'aspects de Wie ich

26. - « So weit oben im Berg und so weit von der nächsten Straße hat man nicht das Gefühl, dass dieser Ort etwas mit dem Rest der Welt zu tun hat », p. 7.

27. - «In den Bergen. Ganz weit oben. Dort wo schon lange kaum ein Mensch mehr ist. Dort, wo dich keiner fragt, wer du bist. [...] Dort musst du hin. Dort ist dein Platz. Dort bin ich auch bei dir. Guck mal in den Stall. », p. 136. 
mir das Glück vorstelle nous semblent autoriser un rapprochement avec le Schelmenroman, notamment avec le Simplicius Simplicissimus de Grimmelshausen ${ }^{28}$. Un premier parallèle réside bien sûr dans la similitude des contextes, dans l'évocation d'une période de conflit troublée et confuse, avec de nombreux belligérants, associée à la description de territoires ravagés par la guerre. Lévocation de la «ville des ponts » peut ainsi être rapprochée de celle de la ville de Hanau: «J'entrai, mais je ne découvris pas âme qui vive. Par contre les ruines étaient semées çà et là de cadavres $[. ..] \gg^{29}$. Un autre point commun concerne le dispositif narratif avec, dans les deux cas, un personnage principal qui est en même temps le narrateur à la première personne et qui décrit ce qu'il voit avec un regard innocent, à la manière d'un enfant. Ni Viktor ni Simplicius en effet ne donnent d'explication sur les causes ou les enjeux du conflit, ils décrivent simplement ce qu'ils voient. À la suite de la description de Hanau, Simplicius note ainsi par exemple: «Cet affreux spectacle m'inspira une grande terreur, mon esprit simpliste ne pouvait imaginer quel malheur avait bien pu mettre la localité dans un tel état »30.

Un certain nombre d'éléments du récit peuvent en outre être mis en parallèle. Viktor et Simplicius sont tous deux séparés très jeunes de leur famille par la guerre. Le héros de Grimmelshausen a en effet une dizaine d'années lorsqu'il se voit contraint de quitter la ferme de ses parents, brûlée et pillée par les soldats impériaux. On se souvient que Viktor et sa famille ont été chassés de leur appartement et que Viktor s'est, par la suite, retrouvé seul dans la ville, avant d'être recueilli par des religieuses tandis que Simplicius l'est par un ermite qui vit seul dans une forêt. Après cette période caractérisée, pour l'un comme pour l'autre, par une certaine sécurité, s'ensuit, pour les deux protagonistes, une période d'errance. Tous deux survivent tant bien que mal, au hasard des rencontres. Différents détails significatifs nous semblent renforcer encore le jeu intertextuel. On apprend en effet, lors de l'interrogatoire de Simplicius par le gouverneur de Hanau, que le jeune garçon possède un petit cahier en écorce de bouleau dans lequel il écrit et dans lequel

28. - Nous citerons l'édition parue chez dtv (München, 1975) en abrégeant son titre en $\mathrm{S}$ et en indiquant ensuite la partie, le chapitre et la page. On pourrait également bien sûr faire de nombreux parallèles avec Le Tambour de Grass.

29. - « Ich ging hinein, konnte aber keines lebendigen Menschen gewahr werden, hingegen lagen die Gassen hin und her mit Toten überstreut [...] » I, 19, p. 54. La traduction citée est celle de Maurice Colleville (Paris, Aubier, Montaigne, 1963).

30. - «Dieser jämmerliche Anblick war mir ein erschreckliches Spektakul [...], meine Einfalt konnte sich nicht ersinnen, was für ein Unglück den Ort in einen solchen Stand gesetzt haben müßte » (S I 19, p. 54). Interrogé par l'ermite sur ce qui lui était arrivé, il lui avait expliqué que leur maison avait été incendiée par des « hommes de fer » (S I 8, p. 27), il ignore en effet tout de la guerre et ne connaît par exemple pas le mot « soldat». 
il garde la lettre de l'ermite entre-temps mort de vieillesse. Cela ne peut manquer de rappeler le cahier de Viktor et la lettre-testament de sa grand-mère qui sont, avec le crayon, les seuls objets que Viktor garde quand il quitte la ville, une fois ses compagnons de survie disparus (S I 20, p. 58). On peut penser aussi au tambour qui est confié à Simplicius une fois qu'il est devenu soldat du roi de Suède parce qu'il est trop petit pour porter un mousquet (S II 4, p. 108). D'une manière un peu similaire, Viktor reçoit en effet également un «tambour» (en réalité une casserole avec deux cuillers) après avoir trouvé sous un lit un fusil trop lourd pour lui qu'il recache aussitôt, car il a probablement le sentiment de s'intéresser à quelque chose d'interdit ${ }^{31}$.

Outre ces éléments, les deux romans semblent avoir aussi en commun, en contre point à l'évocation d'une réalité extrêmement dure, la référence à d'autres mondes, autrement dit à des modèles utopiques. On peut citer dans Simplicius Simplicissimus celui de Jupiter (S III 3-6) qui évoque un monde de paix sans différends religieux, celui que représentent les habitants du lac de Mummel qui vivent dans un monde sans passion, sans douleur et sans péché (S V 12-17), ou encore celui décrit par les anabaptistes hongrois (S V 19) ${ }^{32}$ qui ne connaît ni exploitation ni misère ni pauvreté. Et on peut penser en parallèle, dans le roman de Kordic, au chapitre qui donne précisément son titre au livre et qui contient la manière dont Viktor se « représente le bonheur» (165-170) en évoquant, comme on l'a vu, sous les traits d'un groupe d'éléphants, l'image d'une grande famille unie qui n'oublie pas ses morts et où les enfants découvrent le monde qui les entoure, une nature hospitalière dans laquelle les différents animaux vivent en harmonie.

L'hypothèse intertextuelle peut peut-être également contribuer à éclairer la fin du livre et la présence du prologue. Le roman de Grimmelshausen se termine en effet, comme on le sait, par un « adieu au monde » (S V 24): Simplicius, qui au fil de ses aventures, a découvert à quel point le monde est faux, mauvais et injuste décide de le quitter et de redevenir ermite. Le dernier chapitre du livre de Viktor, intitulé «Le dernier jour», décrit «le garçon » qui, arrivé au bord de la mer, décide de quitter le monde terrestre et de s'enfoncer dans l'eau ${ }^{33}$. Et le livre se termine sur une phrase quelque peu énigmatique où l'on voit sa «dernière pensée » «disparaître pour toujours dans la lueur des

31. - Tous ces éléments seraient bien sûr à mettre en parallèle avec Le tambour de Grass, qui, comme on le sait, est largement inspiré par le roman de Grimmelshausen.

32. - Nous renvoyons pour plus de précisions aux réflexions de Volker Meid, Grimmelshausen, Stuttgart, Reclam, 2011, p. 63-71.

33. - Si le récit est le plus souvent rédigé à la première personne, certains passages sont à la troisième personne, comme si le narrateur se dédoublait, ce qui pourrait être le cas à la fin du texte où un avatar de Viktor disparaîtrait tandis que l'autre se confondrait avec l'ermite du prologue. 
étoiles $»^{34}$. Après le mot « fin », on trouve cependant encore une photo représentant une maison qui pourrait ressembler à la maison familiale du «village des gens heureux » où est né Viktor ${ }^{35}$, si bien qu'on aurait également chez Kordic une structure circulaire avec un retour au point de départ, associée à l'évocation d'un personnage d'ermite tel que celui qui s'exprime dans le prologue.

Si la référence au conte nous semble donc pertinente pour caractériser certains des aspects de l'écriture de Kordic, elle ne nous paraît pas suffisante pour rendre compte des références intertextuelles à l'œuvre dans le roman. Le parallèle avec le roman picaresque, en particulier avec le début et la fin de celui de Grimmelshausen, nous semble au moins aussi pertinent (écriture à la première personne, naïveté du héros, composition en épisodes liés aux rencontres du personnage, structure circulaire, présence d'éléments utopiques), à la réserve près que la dimension critique nous semble moins nettement présente dans Wie ich mir das Glück vorstelle que dans Simplicius Simplicissimus.

34. - « Wie ein Feuerwerk [...] steht Viktors letzter Gedanke in tausend kleinen Funken weit über dem Meer und verschwindet kurz darauf für immer im Leuchten der Sterne. », p. 170.

35. - Richard Kämmerlings qui a interviewé Martin Kordic (cf. infra note 3) affirme en tout cas que cette maison est celle d'un oncle de l'auteur: « Heute ist er [Martin Kordic] mehrmals im Jahr dort [in der Heimat seines Vaters], wohnt dann bei einem Onkel in einem Bauernhaus am Dorfrand. Im Prolog und im Epilog des Romans kommt der Hof vor - als paradoxes Signal der Beglaubigung einer ganz fiktiven, manchmal magisch-realistischen Geschichte ». 